\title{
An outline of some future research issues for internet pragmatics
}

\author{
Francisco Yus
}

\begin{abstract}
Internet communication has evolved a lot since it first became popular in the early nineties of the last century. Pragmatics has also evolved and has tried to come to terms with the non-stop changes that internet is constantly producing in our lives and especially in how we communicate and interact. We are probably now at a stage of internet development in which we can make some sound predictions regarding certain challenges that a pragmatics of internet communication will have to face in the next few years to deal with the radical changes that are taking place in today's internet use. This article will be devoted to listing some of these research issues and to discussing what pragmatics can do to address them accurately, ranging from those issues centred upon the interpretation of online discourses to those involving interfaces and their options for contextualisation.
\end{abstract}

\section{Introduction}

In previous research (e.g. Yus, 2015a, 2016a), up to five stages of relationships between online and offline identities were proposed, which also reflect the evolution of internet's presence in our lives. In a nutshell: at the first stage, at the beginning of the 1990s, internet was limited in information and offline scenarios were the main source of identity shaping. At a second stage, at the beginning of the 2000 s, users played with the duality of offline-online interactions. In a mainly text-based form of communication, internet was useful to mask macrosocial attributes (such as sex, race) as well as visual identity attributes such as the user's body, face, racial origin, etc., while nickname-based identities multiplied. At a third stage, deeper into the 2000s, two parallel and converging processes were witnessed: a growing virtualisation of offline areas of interaction (streets, bars...) and a growing materialisation of online spaces for interaction (internet fora, virtual communities, social networking sites...). The fourth and fifth stages are similar. Basically, these reflect the fact that users have developed an ability to switch from offline to online networks of friends with different degrees of commitment and intensity depending on what portion of their identities is shaped discursively on the Net, ranging from those who rarely log on, to those who reject their physical identities entirely and can only "be themselves" on the Net. At the fourth stage, often users no longer differentiate between the strength of online and offline environments. And in the fifth phase, within today's use of the Net, there is a presumption of physical-virtual congruence, since users do not turn into different people in either of the environments (offline/online), as happens with self-presentation on Facebook compared to self-presentation in physical spaces. As Zhao et al. (2008: 1819-1820) correctly state, "users regard their online presentations as an integral part of their overall identity production and seek to coordinate their online identity claims with their offline selfperformance."

Pragmatics, and specifically internet pragmatics, have also evolved to accommodate new forms of virtual communication, and now there are many publications addressing the constant changes that internet is producing in how we interact and the role of interfaces in the quality and quantity of these interactions (Yus 2018a). And we are now at a stage of development in internet pragmatics in which we can predict certain research trends and issues that will be important in the near future. This paper is devoted to commenting on a number of these research issues. Needless to say, the list is not exhaustive but mainly illustrative, the future might develop in different directions, and new kinds of interactions and interfaces may appear in the future that defy our conceptualisation of communication as typically addressed by internet pragmatics 
nowadays.

\section{What is said versus what is meant (and interpreted)}

Internet pragmatics has produced a large number of publications analysing varied forms of internet communication. Among many others, several may be mentioned, including Flores Salgado et al. (2018) on politeness in WhatsApp, Lutzky and Gee (2018) on commenting blog entries, Scott (2018) on a pragmatics of hashtags, Grundlingh (2018) on memes as speech acts, Morrow (2017) on advice and requests on social networking sites, Paulus et al. (2016) on conversation analysis applied to online talk, Schubert (2017) on cohesion on social media, McKeown and Zhang (2015) on email openings and closings, Dayter (2016) on speech acts in microblogs, and Placencia et al. (2016) on complimenting on Facebook.

In most of these publications, what is typically done is to use a solid body of research within pragmatics (theories, approaches, models...) made on offline communication and interaction and to check the extent to which new forms of communication and interaction on the internet fit this research. An example is Scott (2018): she uses the relevance-theoretic distinction between conceptual and procedural meaning ${ }^{1}$ and shows how Twitter hashtags often fit the role of the latter. However, in future internet pragmatics research, it would be interesting to consider a different approach, namely how new forms of discourse, communication and interaction on the internet trigger adjustments, extensions and a re-shaping of research within internet pragmatics in order to fit new emerging forms of internet communication.

In any case, as is claimed within cyberpragmatics (Yus 2011), the characteristics of the different interfaces or applications for communication (chatrooms, WhatsApp, email, web pages, etc.) affect the quality and quantity of contextual information accessed by users, the mental effort devoted to interpretation, and the very choice of an interpretation. Their "material qualities" (i.e. their affordances) will have an impact on how relevant or irrelevant the eventual interpretation is. Similarly, these also have an impact on how internet pragmatics addresses virtual communication. Take, for instance, utterances on mobile instant messaging. In general, concerning face-to-face interactions, pragmatics claims that there is an informative gap between what the speaker intends to communicate and what he/she literally says, and another one between what is literally said and eventually interpreted, gaps which have to be filled by inference with the aid of context.

In text-based online conversations such as those in mobile messaging, these gaps also exist, but with differences. In a face-to-face conversation, there are informative gaps between what the speaker intends to communicate (for example (1a)) and what the speaker literally says (1b), which are related by resemblance, rather than being exact copies, and a similar gap between what the addressee hears (1b) and what he/she finally interprets, (1c), which involves locating referents for the proper name and the pronoun, together with an adjustment of the concept "drink," which is narrowed into "alcoholic drink" (see also the analysis of concept adjustment in examples (6-7) below). However, since users typically engage in text deformation or text oralisation (repetition of letters, creative use of punctuation marks, emoji...), further gaps turn up

1. As was summarised in Yus (2010), according to relevance theory (Sperber and Wilson 1995), the human cognitive system infers two basic types of information encoded by linguistic expressions: concepts or conceptual representations on the one hand, and procedures for manipulating them on the other. Connectives such as but should not be seen as encoding concepts, but as procedural devices which constrain the inferential phase by indicating the kind of cognitive process that the hearer should go through. In the case of but, for example, the hearer is alerted to a contrast to what was stated before the connective (hence reducing the eventual overall effort). 
which also have to be filled, this time regarding (2b) and (2e), that is, what the users could have said in an oral conversation resembles what the user intends and finally types, and what the users could have heard in an oral conversation resembles what the user reads and finally interprets. This is so because very often users type their oralised texts with a face-to-face version of their utterances in mind which they try to imitate textually by using oralising techniques. ${ }^{2}$

(1) a. What the speaker intends to communicate resembles...

"I am worried about Tom Smith. Tom Smith and his wife Sarah drink too much alcohol."

b. ...what the speaker actually says, which resembles...

"I am worried about Tom. He and his wife drink too much."

c. ...what the addressee interprets (in successful communication):

"I am worried about Tom Smith. Tom Smith and his wife Sarah drink too much alcohol."

(2) a. What the sender user intends to communicate resembles:

"Shall we meet next Friday, have supper, a drink and go partying?"

b. What the sender user could have said (face-to-face), which resembles:

"Shall we meet next Friday, have supper, a drink and go partying?"

c. What the sender user actually types, which is the same as:

"Friday [emoji of bowl of pasta] [emoji of pot of beer] [emoji of dancing woman]."

d. What the addressee user literally reads/interprets, which resembles:

"Friday, a bowl of pasta, a mug of beer, a woman dancing."

e. What the addressee user could have heard (face-to-face), which resembles:

"Shall we meet next Friday, have supper, a drink and go partying?"

f. What the addressee user actually interprets:

"See you next Friday, have pasta together, drink a beer and go dancing?"

\section{Application of pragmatic research to multimodal discourses}

Nowadays, communication draws extensively on textual, visual and multimodal discourses, often tending to highly multimodal compositions in which text and image collaborate in conveying the intended interpretation. As is summarised in Yus (2018b), multimodality is becoming increasingly important nowadays due to the pervasiveness of media discourses that combine different modes of communication (text, picture, audio, video...). A basic definition of multimodality is suggested by Stökl (2004: 9), for whom "multimodal refers to communicative artefacts and processes which combine various sign systems (modes) and whose production and reception calls upon the communicators to semantically and formally interrelate all sign repertoires present." For van Leeuwen (2015: 447), the term "indicates that different semiotic modes (for instance language and picture) are combined and integrated in a given instance of discourse or kind of discourse." In fact, the most interesting picture-text combination for a pragmatic (i.e. cyberpragmatic) analysis is the one in which the eventual meaning cannot be

2. It should be noted that in this text-based dialogue involving (2a-f) there is a slight misunderstanding: The sender user resorts to the emoji of a bowl of pasta, the mug of beer and the dancing woman as metonymies of "have supper", "have a drink" and "go partying" respectively. By contrast, the addressee user interprets the three images literally as meaning "have pasta", "drink a beer" and "go dancing." 
obtained from the partial meanings of text or picture taken separately, but only from their combined meanings that yield implications (see below).

Finally, Jewitt (2016) summarizes the key assumptions in studies of modality: (a) all communication is multimodal; (b) analyses focused solely or primarily on language cannot adequately account for meaning; (c) each mode has specific affordances arising from its materiality and from its social histories which shape its resources to fulfil given communicative needs; and (d) modes concur together, each with a specialized role, to meaning-making; hence relations among modes are key to understand every instance of communication.

On the internet, very often text and picture influence each other so that the eventual interpretation conveyed cannot be obtained from the partial meanings of text and image taken separately, but only from the combination of both to yield new non-literal interpretations of their referents. In Yus (2018b), for instance, this mutual cooperation of text and image to yield an interpretation that cannot be obtained from the meanings of either text or image independently can be seen in a series of memes of the image macro type (lines of text at the top and bottom of the meme and an image in the middle) that were analysed in their multimodal quality, all of them sharing the same picture of two girls laughing and the interpretation of the texts, invariably related to sex role-connoted information, becomes immediately reversed when text and image are combined, as in (3-4):
(3) Top text:
And then I said...
Image:
Two girls laughing.
Bottom text: I don't care about your money, I love you for who you are.
(4) Top text: $\quad$ And then I said...
Image: $\quad$ Two girls laughing.
Bottom text: I'll be ready in 5 minutes.

In the future, we will witness an increasing application of pragmatic theories to this kind of visual or multimodal communication, a transference of analysis from the typical cues-filtered, text-based interaction to a more adequate verbal-visual or multimodal object of research. Take the explicit/implicated distinction, for instance. Recent developments in pragmatics have shown how context is needed for obtaining both the explicit and the implicated interpretation, even if the former had been dismissed in previous research (e.g. by Grice). Consider the example in (5):

(5) Max: How was the party? Did it go well?

Amy: There wasn't enough drink and everyone left early.

According to Grice's (1975) famous coinage of implicature, Amy clearly implicates that the party did not go well (which Max obtains by pairing Amy's utterance with contextual information, specifically commonsense encyclopaedic information on parties). However, he dismissed the importance of context in obtaining the explicit interpretation of Amy's utterance (what is said, in his terminology, basically restricted to reference assignment and disambiguation). By contrast, recent developments in pragmatics (e.g. relevance theory) show how the hearer also has to rely on context to obtain an adequate explicit interpretation or explicature. In the case of (5), drink is adjusted as specifically meaning "alcoholic drinks," everyone is narrowed into "everyone at the party," and the concept early is interpreted against the cultural background of what is commonly assumed to be "early" for parties in Max and Amy's society.

In this sense, in several publications it is claimed that the explicit/implicated distinction is 
also applicable to visual and multimodal discourses, whose comprehension yields visual explicatures and visual implicatures (two terms related to the denotative/connotative distinction in traditional visual studies), in the sense that the conceptual referent associated with the image is similarly inferred, even if coding and decoding differ in texts and images (see Yus 2008, 2016b; Forceville and Clark 2014). Therefore, in the future we will doubtless see pragmatic studies that take into account at least six inferential procedures for online multimodal discourses (Yus 2018b):

Strategy 1. To decode and inferentially enrich the verbal content of the multimodal discourse in order to obtain the explicit interpretation of the text or explicature.

Strategy 2. To derive implicatures from verbal content, if these are necessary to reach a relevant interpretation of the verbal content of the multimodal discourse.

Strategy 3. To decode and inferentially enrich the picture to yield a visual explicature.

Strategy 4. To derive implicatures or implications from the picture in the multimodal discourse, if these are necessary to reach an adequate interpretation of the discourse as a whole.

Strategy 5. To infer possible combinations of text and picture to yield interpretations (typically implicated ones or verbal-visual implicatures) that are only possible from the combination of these sources of information (text and picture) and not from either of them taken separately.

Strategy 6. To access as much contextual information as necessary to obtain interpretations out of strategies 1-5 above.

These strategies are not meant to be sequential or successive. Besides Strategy 6, which is pervasive throughout the other five, even if the author of the multimodal discourse has a specific verbal-visual reading path in mind when creating the discourse, there is no guarantee that these strategies will be followed in a similar way across users, and this may affect the eventual satisfaction or correct interpretation of the multimodal discourse.

Another application of text-centred pragmatics to visual and multimodal discourses is the relevance-theoretic notion of concept adjustment. In a nutshell, on many occasions (if not all), the concept that underlies a coded word in an utterance is adjusted pragmatically, in such a way that the prototypical concept associated with that word (as one would find in a dictionary, for example) is not relevant enough and therefore it has to be adjusted and so is different from (i.e. it only resembles) the concept actually intended and communicated in a context (called ad hoc concept). This communicated concept may be narrower than the coded concept, as in (6), or broader, as in (7). In all of these cases, the ad hoc concept that is eventually communicated (DRINK*, EMPTY* $)^{3}$ only resembles the concept literally coded in the utterance.

(6) I am worried about Jim... He drinks too much.

[specifically, Jim drinks too much alcohol].

(7) We entered the pub but we left since it was empty.

[not literally empty; rather, with few people, including the waiter].

My point is that the same idea of concept adjustment may be applied to visual discourses, this time rewritten as visual referent adjustment. One example is the use of emoji depicting facial expressions exchanged on mobile messaging applications. The reader normally sees a fixed schematic portrayal of this expression but has to adjust its decoded meaning into a more appropriate ad hoc visual referent. Take the simple emoji of a smile. The reader will match this

3. Adjusted concepts are conventionally represented with an asterisk after them. The encoded concept EMPTY is represented as EMPTY* when understood in context. If there is a need to distinguish two ad hoc concepts of the same encoded word, then two, three or more asterisks are added. 
emoji with its prototypical visual referent: "to be happy." But depending on the textual context in which the emoji is embedded, the reader will have to adjust this referent and eventually infer different (though resembling) ad hoc referents which may be broader or narrower than the coded visual referent.

Another example is the frequent series of memes in which the image depicts a single facial expression or gesture and the accompanying text in the meme forces the reader to engage in a visual referent adjustment in order to obtain an appropriate interpretation of this facial expression, as is the case in the series of memes depicted in Figure 1. The prototypical gestures depicted in the pictures (the little child's gesture and hand movement) are interpreted, in general, as the child being proud of having achieved some goal. However, this default, prototypical interpretation has to be adjusted so as to infer correctly the kind of feeling enacted by the actions described in the text that are placed next to the same picture in those memes. Hence, the visual referent inferred for the gesture will be different when accompanied by the text Finished homework in class (more like "sheer pride"), or by the text Divorced parents. Twice the Xmas presents (roughly "intense joy"). In all of these instances, the user has to adjust the referent of the gesture according to the accompanying text, even though the gesture remains the same.
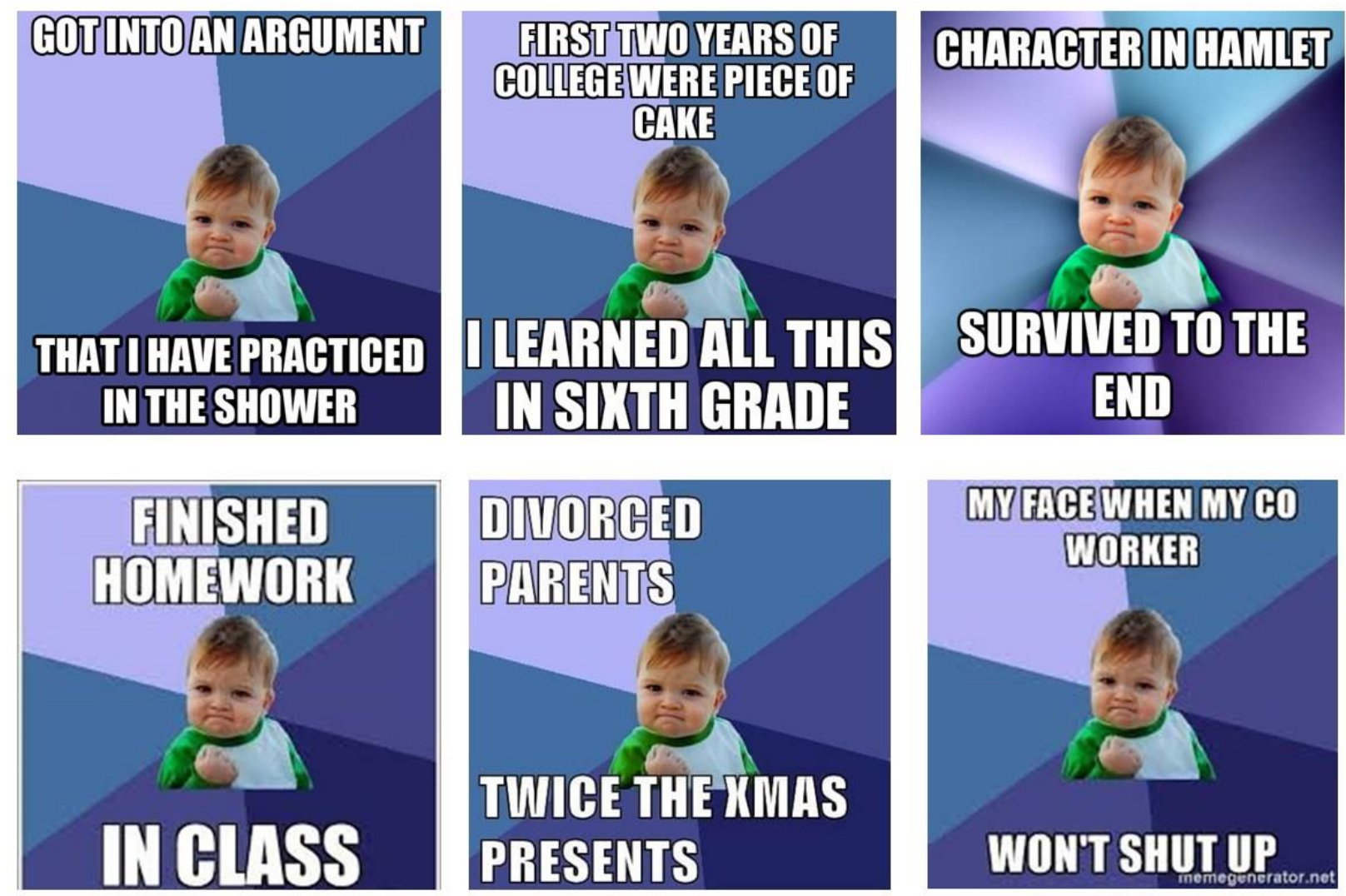

Figure 1. Ad hoc visual referent adjustment in a series of memes.

\section{Typed messages with visual support}

Nowadays, text-based communication is still very frequent, as in the extensive use of mobile messaging applications (WhatsApp, WeChat, Line, Snapchat...). However, users constantly "enrich" typed text with text deformation (Yus 2005), also called oralised written text (Yus 2011). Crucially, all kinds of visual discourse also accompany the text, interacting with it and generating varied interpretive outcomes. Visual aids include emoji (as is illustrated in Figure 2), 
photos, videos and animated GIFs, among others. Internet pragmatics will have to deal with these extensively used innovative discursive combinations, and analyse their interpretive, personal and social implications. As can be seen in the Figure, users constantly complement text with visual discourses with a number of possible underlying intentions, ranging from mere saving time while typing, to enhancing, masking, contradicting, adding emotional connotations and so on to the accompanying texts.

As was claimed in Yus (2014), text deformation and the use of emoji always communicate additional information related to propositional attitudes, feelings, emotions, determination or ironies, joking, etc. ${ }^{4}$ And their analysis will remain a central issue of internet pragmatics. Take the conversation in (8) from Sampietro (2016):

(8) Laura: Es hoy la entrevista? Muuuucha suerte [kissing emoji].

[The interview is today? Loooootsa luck].

Lorena: Gracias cariño [smiling emoji] [smiling emoji] [smiling emoji].

[Thanks my dear].
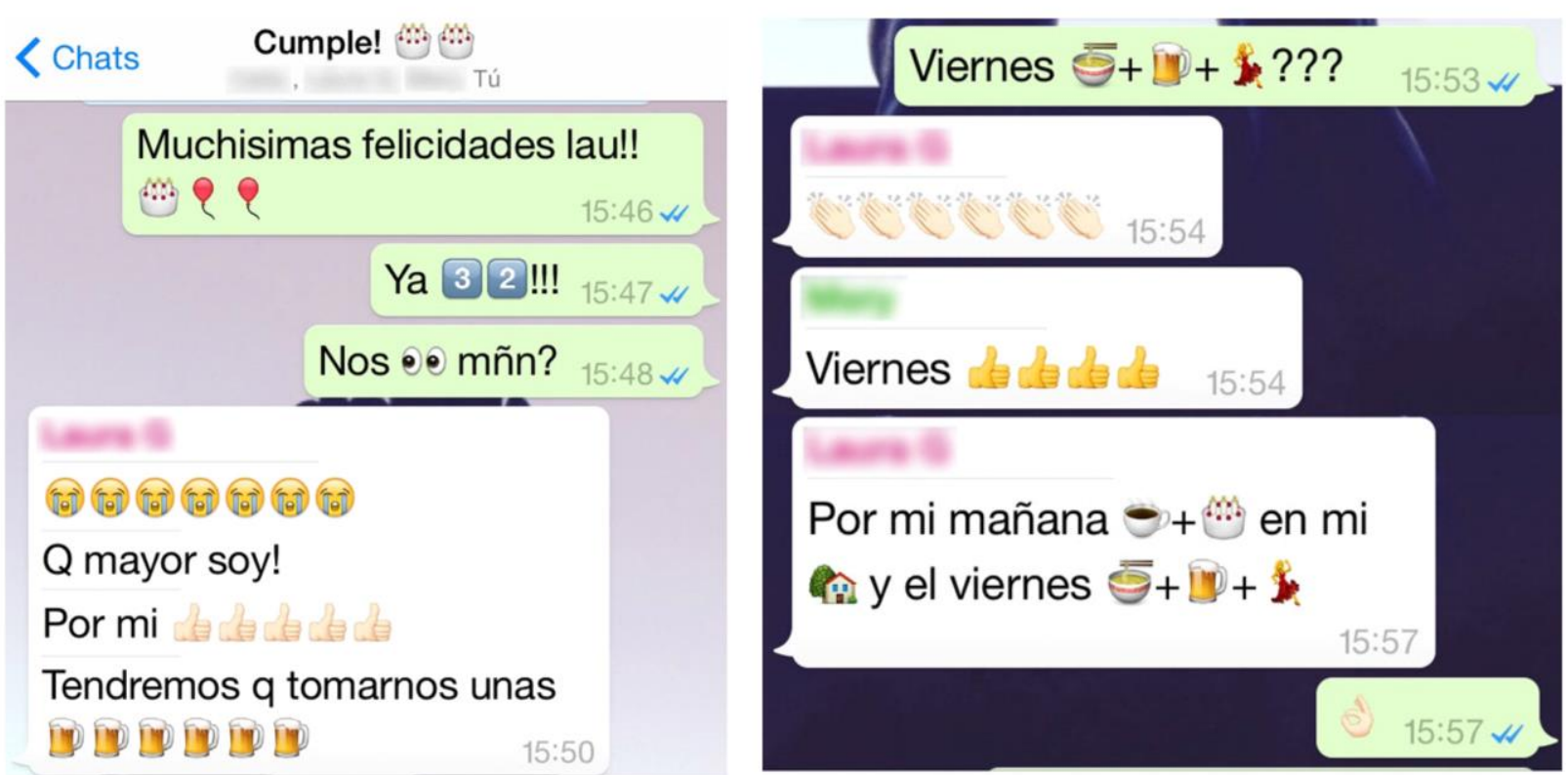

Figure 2. Emoji in an informal WhatsApp conversation.

Internet pragmatics has to account for the additional meanings associated with the visual support of emoji and the repetition of letters in (8). Sampietro's (2016) analysis, regarding Laura's text, shows that the wish of luck is emphasised by text deformation and the repetition of the exclamation mark. The emoji seems to play an independent role, and communicates its own speech act: "to say goodbye." It also substitutes the textual "I give you a kiss" and indicates the desire to end the conversation. Concerning Lorena's text, the repeated emoji intensifies the act of thanking, but it could also be a qualifier of another proposition, such as "I am happy that you remembered to wish me luck."

4. In Wiseman (2013), an eighteen-year-old informant says: "You can tell what she wants pretty much by how she texts. The dry 'Hey' is O.K. But then there's some that have the 'Heyyy' with the extra y's and the winky face, and that means this conversation could possibly go somewhere. They're probably the hooking-up type." This is an example of additional meanings attributed to these discursive strategies. 


\section{Voice-based communication (but not necessarily synchronous and oral)}

Internet pragmatics will have to face the challenge of explaining why users who have highly contextualised options for internet communication, for example free voice and video calls through free mobile applications such as WhatsApp or WeChat, shy away from these synchronous options and prefer more cues-filtered options such as texting or engage in asynchronous voice-recorded files. Turkle (2015) analyses this phenomenon as part of her claim that we have to reclaim face-to-face conversations. Her analysis shows that synchronous conversations are negatively constrained, whereas texting and recorded files provide users with several advantages that lead to an increasing reluctance to engage in synchronous conversations. When Turkle (ibid.) asks "What's wrong with conversation?" she obtains illustrative answers. A young man makes things clear to her: "What's wrong with conversation? I'll tell you what's wrong with conversation! It takes place in real time and you can't control what you're going to say" (a negative contextual constraint in Yus 2017a terminology). Unlike face-to-face interactions, internet provides users with several advantages. Turkle (ibid.) mentions that texting gives more space to say things right and make things right. If one does something wrong they can fix it right away. The phone call is in real time and real time is a place of awkwardness. An informant further acknowledges that "you can put so little effort in when you text and then you get instant gratification. I can connect with fifteen people with no effort and it feels so good to just extend the feelers and get a positive response. I would rather have that than a conversation a lot of the time."

\section{Increasing importance of the physical in supposed-to-be virtual communication}

The mobile phone liberated us from the tyranny of physical space, allowing us to call people from anywhere, but nowadays a number of locative media have brought physical location back to the forefront of users' interactions. In the future, internet pragmatics will often bring into the analysis location-based information (text, images, comments) tagged onto locations by the users themselves.

The term 'locative media' may be defined as those apps or interfaces that enable and focus on the physical location of the user while communicating with peers, friends or acquaintances in a virtual environment. They provide users with a reflexive awareness of the location of themselves and others or their mutual proximity (Licoppe 2013). They encompass mapping services and social media applications that allow for tagging content and mapping information related to the user's physical location (Frith 2015).

Interestingly for internet pragmatics, locative media have generated alterations, not only in our conceptualisation of place and its relationship with the digital information attached to it, but also in the impact of this physical-virtual connection on what interpretation is intended, what information is supposed to be shared among users, what interpretation is selected and whether this interpretation eventually turns out to be relevant or irrelevant in those instances of communication that are mediated by these locative media.

A typical example of location-based communication is the Facebook check-in (Yus 2018c). Apparently, checking in on where one is having a meal or on the fact that one is flying from Madrid to London seems to provide users with little substantial information, but internet pragmatics has to address the possible underlying intentions and predict an eventual (relevant?) interpretation. Certainly, check-ins are not published on Facebook for no reason, and the user 
expects, on most occasions, some inferred meanings regarding that specific location and not others. Possible intentions include (a) establishing a social connection with friends, (b) reviewing location, (c) discovering new places to visit, (d) keeping track of already visited places, (e) fighting boredom, and (f) providing users with relevant information on the place, among others.

Besides, check-ins generate mutuality of information. This mutual information becomes a preliminary context upon which further interactions among users may take place. Schwartz (2015: 95-96) proposes the term documentation of relationship for the action of checking in, because check-ins are often accompanied by photographs and tips that result in a rich documentation of the user's interactions and whereabouts.

Finally, check-ins generate a number of feelings and emotions (non-propositional effects) in both sender and addressee users. As was commented upon in Yus (2018c), check-ins create and maintain a sense of presence and ambient intimacy with friends, as well as generating feelings of membership to one's virtual group or community. These often leak beyond the user's intentions, but may end up relevant anyway. For example, sharing mundane everyday activities may produce ambient intimacy among networked contacts (the same applies to trivial tweets on Twitter). The Facebook check-in would therefore produce a vivid sense of co-presence with the user who is at that particular location.

\section{Varying sources of mutuality in the physical-virtual interface}

One of the basic premises in pragmatics is that there is information which interlocutors assume as shared or mutual in the course of a conversation, but how interlocutors manage and assume this area of mutuality has been an object of academic research for many years. In a nutshell, as summarised in Yus (1998), it is often difficult to determine which information is indeed shared by interlocutors while (or even before) interaction takes place. In other words, how do speakers distinguish the information they merely know from that they really share? Relevance theory argues that as the traditional concept of mutual knowledge is unable to clarify this point, and the concept is equally unable to explain precisely what underlies its definition of mutuality.

Specifically, according to relevance theory (Sperber and Wilson 1995), the aim of communication is not so much to transfer information to other people, but rather to make information mutual to both interlocutors (mutually manifest to both of them, in its terminology). What the person is capable of entertaining mentally in a specific context or situation is called the person's cognitive environment, which varies from one context to another. Information (a set of assumptions in relevance-theoretic terminology) which is manifest to several individuals constitutes their shared cognitive environment. This area of mutuality, again, changes according to different contextual parameters and is made up of mutually manifest assumptions. Communication, then, is a matter of making certain assumptions mutually manifest to both speaker and hearer (Yus 2010: 761).

In the case of mixed face-to-face and virtual interactions, for example interlocutors who chat face to face and, at the same time, text other physically absent interlocutors with their mobile phones, some new areas of mutuality turn up which have to be addressed by internet pragmatics. A possible situation concerning face-to-face versus virtual mutual manifestness is depicted in Figure 3, in which a speaker and a hearer are engaged in a face-to-face conversation and both may also be having virtual text-based conversations with a single interlocutor or a group of interlocutors (as happens with WhatsApp groups, for instance):

First, the speaker and hearer have their own personal cognitive environments, depicted as 
the central bold-lined squares in the middle of the Figure. The intersecting area (A) is the interlocutors' mutual cognitive environment, made up of assumptions which are mutually manifest to both of them.

Second, the speaker and the hearer may also be having a text-based conversation with a single user through his/her mobile phones, for instance by texting on a mobile messaging app. In this case, a mutual cognitive environment exists between the speaker and this single user (B) and between the hearer and that single user (D).

Third, both speaker and hearer may also be engaged in an interaction with a group of people within a mobile messaging app. If this is the case, then there would be a broad mutual cognitive environment between the speaker and the users within that group (C) and between the hearer and his/her own group of text-based interlocutors (E).

Fourth, a possible interactive configuration is the one in which the speaker has a mutual cognitive environment both with a single virtual interlocutor and with the users within a mobile messaging group (F, depicted as a square with ". . . ." lines) while talking face-to-face with the hearer. The same would apply to the hearer and his/her single/group interlocutors (G, depicted as a square with "......" lines).

Fifth, another possibility is that all of the interlocutors involved might share a broad cognitive environment, made up of assumptions that are mutually manifest to all possible interlocutors $\left(\mathrm{H}\right.$, depicted as a square with ". ${ }_{-} \cdot{ }_{-} \cdot$ " lines). For instance, the two interlocutors in physical co-presence might be accessing the same messaging group and those in the group may also know about these interlocutors' presence while interacting as a whole broad group.

And finally, a possibility which has not been reproduced in Figure 3 (for the sake of visual clarity) is the one in which the speaker and the hearer are having a conversation in a physical scenario and, at the same time, both are having a virtual, text-based conversation with the same group of users through a mobile messaging app. 


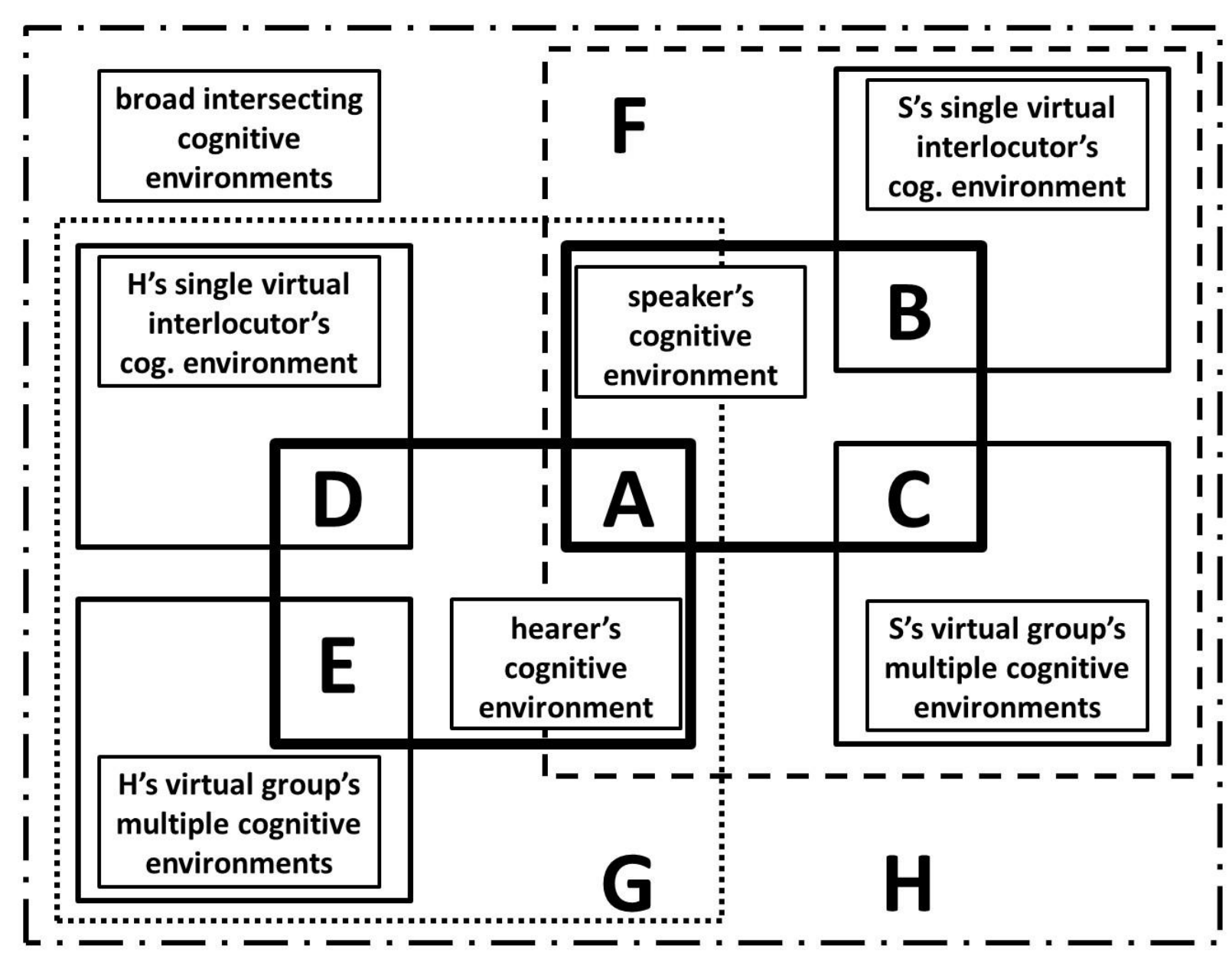

Figure 3. Different levels of mutual manifestness in overlapping physical and virtual interactions.

\section{Decreasing importance of substantive content in exchange for phatic interactions}

Phatic communication has traditionally been regarded as important for sociability but irrelevant for the transfer of substantive content. It is "primarily aimed at establishing and maintaining social bonds between individuals over and above the exchange of information and hence do not necessarily express any particular thought nor aim to exchange facts" (Vetere et al. 2009: 178). Most definitions, one way or another, emphasise the contrast between the lack of interesting information and the eventual "social" relevance of the phatic act of communication (see, for instance, Žegarac 1998: 328).

Phatic communication is very important in order to describe and understand the extent and depth of the interactions by today's internet users through online interfaces, typically devoid of informativeness but nevertheless relevant in the generation of a number of personal and social effects. As Miller (2008: 398) correctly claims in his seminal paper on the so-called phatic culture, "we see a shift from dialogue and communication between actors in a network, where the point of the network was to facilitate an exchange of substantive content, to a situation where the maintenance of a network itself has become the primary focus. Here communication has been subordinated to the role of the simple maintenance of ever expanding networks and the notion of a connected presence." What may be called phatic effects, including feelings of connectedness, bonding, sociability, group membership, etc. certainly make up for the lack of interest that the thousands of virtual messages exchanged on a daily basis objectively possess.

The phatic internet is also pervasive nowadays because the technologies for virtual 
interactions were either initially designed for casual interactions or have subsequently acquired a phatic quality in their design, that is, have become phatic technologies, defined in Wang et al. (2011: 46) as follows: "A technology is phatic if its primary purpose or use is to establish, develop and maintain human relationships. The users of the technology have personal interactive goals." The phatic connotation entails that the essence of these technologies is relationship building, rather than information transfer. As such, relationships and reinforcement of connection demand a constant supply and exchange of messages, typically through mobile phone interfaces (apps). Interfaces such as Facebook, Twitter or WhatsApp not only allow for non-stop interactions that make up for the interlocutors' lack of physical co-presence, but have also implemented phatic affordances in their design (as happens with the Messenger option on Facebook). Apart from the actual spaces devoted to typed interactions, there are interface elements which trigger a phatic feeling of connectedness and presence, for example the "paralinguistic digital affordances" (Carr et al. 2016) on Facebook such as acknowledgements ("like") and calls for attention ("poke"), together with automated linguistic reminders of initiated interactions (personal emails). These phatic elements serve the purpose of keeping users connected and aware of each other's presence.

Internet pragmatics will have to adjust its research interests from the initial default assumption that people exchange substantive and relevant content, to a situation in which information is irrelevant and nevertheless ends up relevant to the users through phatic effects, that is, through non-propositional means. In Yus (in press), for instance, it is claimed that in order to account to what is really at stake in the phatic quality of internet interactions, we have to reformulate our idea of phatic communication. Indeed, pragmatic theories such as relevance theory address phatic communication as (a) involving an intentional act; (b) centred upon the inference of information of a propositional kind; (c) exhibiting degrees (utterances may be phatic to a greater or lesser extent); (d) focusing on the speaker and his/her willingness to interact or socialise; and (e) transferring information which is objectively irrelevant or devoid of interest, but relevant in the effects it exerts on relationship, bonding or socialisation, and these effects are shaped as weak implicatures.

Although this is undeniably the case on some occasions, in Yus (ibid.) it is claimed that phaticness may exhibit other possibilities, and that these added possibilities are particularly useful to understanding the extent and depth of phatic communication on the Internet, and that phaticness (a) may be generated in addition to what was specifically meant with an intentional act of communication; (b) may possess a non-propositional quality, in the shape of feelings, emotions, desires that are attached to the initially irrelevant (propositional) content communicated; (c) may also be obtained from non-phatic utterances; (d) may be addresseecentred beyond the addresser's intentions; and (e) may be generated out of fully relevant content.

\section{Elements that frame virtual communication (contextual constraints)}

In the future, internet pragmatics will have to pay attention to what in previous research (e.g. Yus 2017a) were called contextual constraints. In a nutshell, internet communication is affected by a number of interface-related and user-related qualities that may alter the eventual estimation of the relevance of the act of communication and affect its interpretive outcome. These are mainly related to the interface's affordances and usability, the users' management of the interface, the kind of relationship existing between interlocutors, the user's personality, the user's task upon logging onto the Net, etc. The term is restricted to aspects that underlie the acts of communication and the users' interactions (i.e. they exist prior to the interpretive activity) and 
constrain their eventual (un)successful outcome. They frame, as it were, communication and have an impact not only on the quality of interpretation, but also on the willingness to engage in sustained virtual interactions. Needless to say, contextual constraints exist in every act of communication, not only internet-mediated ones, but their influence is much more noticeable on the internet, where interactions are often devoid of physical co-presence and utterances often exhibit a cues-filtered quality. In any case, they exist prior to the interaction and hence they would appear not to be an inherent object of pragmatic research, but their role in the outcome of communication makes its analysis relevant to determining why communication on the Net turns out satisfactory or fruitless.

\section{Increasing importance of non-propositional effects (feelings, emotions)}

Pragmatics has been reluctant to include non-propositional information in the analysis of human communication, ${ }^{5}$ but this information covering feelings, emotions, impressions, etc. plays a crucial role in internet communication (as well as in offline face-to-face communication). Therefore, they should be incorporated into internet pragmatics research in the future. As is described in Yus (2018d), the range of communicative possibilities involving feelings and emotions, which can be grouped under the umbrella terms affective attitude (when intentional) and affective effect (when "exuded" or "leaked" unintentionally"), are varied if we take into account axes such as propositional/non-propositional quality or acted upon/non-acted upon, together with the initial distinction between intentional and unintentional transfer of affective information.

The term "acted upon" refers to the fact that feelings and emotions may be produced without the individual intervening in their extent and intensity, whereas on other occasions there is conscious control on their production, specifically regarding the fact that feelings and emotions may be produced without control in the users or they may be fully aware of these nonpropositional effects and "act" on them as part of the intended interpretation or the eventual interpretation obtained within/beyond the sender user's intentions. As Langlotz and Locher (2013: 91) summarise, while some cues may result from spontaneous expressions of genuine emotional states, other cues may be used strategically to emotionalise a given message in the

5. As Sperber and Wilson (1995: 57) explain, there is a very good reason to assume that what is communicated is propositional: it is relatively easy to say what propositions are, and how inference might operate over propositions. No one has any clear idea how inference might operate over non-propositional objects: say, emotions. "Propositional contents and attitudes thus seem to provide the only relatively solid ground on which to base a partly or wholly inferential approach to communication." A reviewer of this paper asked me to be more specific about why communication should be propositional. He/she adds that "there are a number of approaches to communication which conceive communication as conveying propositional meaning, interpersonal meaning and interactional meaning -with every communicative contribution being composed of all three parts (e.g. interactional sociolinguistics, discourse pragmatics." However, interpersonal and interactional meaning have also been conceptualised as "propositional" in pragmatics, and it is only now that pragmatics and specifically relevance theory have started to account for non-propositional information in pragmatics terms. An example is Wilson's (2017) paper "Communication, comprehension and 'non-propositional' effects," in which she proposes a relevance-theoretic account of non-propositional information.

6. Unintended leakage of affective effects is crucial nowadays. In previous research, the term non-intended nonpropositional effect was proposed, referred to feelings, emotions, impressions, etc. which are not overtly intended by the "sender user," but are nevertheless generated from the act of communication, and add (positively or negatively) to the cognitive effects derived from the interpretation of propositional content. These effects are important for eventual relevance, since they have an impact (a) on the positive/negative outcome of acts of communication; (b) on the preference for a specific site, medium or channel; (c) on why certain interactions are (un)profitable despite the lack of/existence of interesting information; and (d) on how interactions make users feel. 
absence of actual arousal. This entails degrees of control on this continuum from spontaneous to strategic emotional display. Van Kleef (2016: 57) adds that people express emotions to various degrees. At one extreme, there are experienced emotions expressed in an uncensored way, so that the interlocutor gets full insight into the individual's feelings. At the other extreme, we may find suppressed expression of any experienced emotion, to the extent that users' nonverbal or verbal expressions provide no clues as to their internal feeling states.

Initially, if we take into account these axes, several possibilities may be listed (Yus 2018d):

1. Intentional/propositional. The addresser intentionally produces a propositional message whose content conveys his/her affective attitude.

2. Intentional/non-propositional/acted upon. The addresser intentionally conveys affective information non-propositionally, either nonverbally (gestures, vocal expressions) or attached to a verbal proposition (via intonation, gestures in parallel to verbal communication, etc.). Being aware of the affective information that should be conveyed, he/she acts upon this nonpropositional act by enhancing it, reducing it, masking it, etc.

3. Intentional/non-propositional/not acted upon. The addresser intentionally conveys affective information non-propositionally, either nonverbally or attached to a verbal proposition. However, he/she does not feel the need to act upon this non-propositional act by enhancing it, masking it, etc.

4. Unintentional/non-propositional/acted upon. Beyond the addresser's intentions, some information exudes or leaks from him/her and conveys his/her feelings or emotions. The addresser becomes aware of this leakage and acts upon its production by minimising it, masking it, etc.

5. Unintentional/non-propositional/not acted upon. Beyond the addresser's intentions, some information exudes or leaks, thereby conveying his/her feelings or emotions. The addresser does not feel the need to act upon its production, by minimising it, masking it, etc.

\section{Big data and their impact on users' interactions}

Big data, namely algorithmic computations by computer systems monitoring the user's activity on the Net, have an impact on what content is accessed by users, on how often information on other users is accessed (e.g. on Facebook) and on the quality and quantity of users' interactions. As such, they play a part in internet communication, for example in how much information is shared between interlocutors in virtual interactions (see Section 6 above). And in the future, internet pragmatics will have to add the analysis of big data to its research objectives.

In Yus (2017b), some space is devoted to what may be roughly labelled system-to-user communication. The computer systems that control the interfaces for human activity on the Net learn from this activity, store a personal profile of the user's likes, dislikes, preferences, etc. and automatically propose specific sources of information when they are potentially relevant to the user. The default criterion is that a user who has accessed a certain kind of website, bought certain items on the Net or surfed through certain web pages will invariably find it rele vant to be offered information of a similar nature.

Although this is not human-to-human communication, and hence not a proper object of study for internet pragmatics, this automatic assessment of sources of interest by computer systems does resemble relevance-seeking coding/inference of information between people. Indeed, the system attracts the user's attention and alerts him/her that there is certain information that is worth attending to (the system's algorithm automatically computes that it will invariably yield informational benefits to the user regardless of the effort invested). This calling for 
attention plays a similar role to the communicative intention underlying interactions between humans. In this case it is the system that plays the role of an individual calling the addressee's attention to prospectively relevant information. And the system takes full responsibility for what is bound to be relevant to the user, to the extent that the system even stops users from accessing certain information if the computer algorithm decides that this information should not be relevant enough for them to process. An example is Facebook; it automatically filters information in three different ways (Pariser 2011: 37-38): (1) By affinity. The friendlier you become with somebody and the more someone visits your profile and engages in conversations with you, the more likely it is that Facebook will show updates from that user. (2) By the relative value of content. For example, updates on user status, e.g. on the user being no longer married etc., are more valuable and the computer system emphasizes them, but other types of content are also underlined by the system if it detects the user's tendency to see that kind of content. (3) By time. Obviously, the most recently published entries have prominence over older entries.

Other automatic filtering of information by the system includes online bookshops' personalized suggestions depending on the user's previous purchasing activity on the site. And of course, we can also include here the output results by search engines such as Google, which is so popular because it uses algorithms to (apparently) yield the most relevant results. This search engine learns from users and yields personalized results for each user, to the extent that specific users with specific search histories will get radically different output results.

Since computer systems control which information is accessed and even which people users interact with, big data are bound to be an interesting research issue or at least a complement to future research within internet pragmatics.

\section{Pragmatics of virtual agents}

Nowadays, computer assistants and virtual agents are increasingly entering our daily lives. These include mobile phone assistants such as Siri and electronic home devices such as Alexa. These have an amazing capacity to access information on the Net and come up with supposedly relevant answers to the user's queries. However, they are still in their infancy in such pragmatic issues as the interpretation of humour, the determination of ironic intentions or the ability to access specifically the amount of contextual information that the user intends as part of his/her intended explicit and/or implicated interpretations. In the near future, though, analysts will probably become interested in how to "train" these devices to become more "pragmatic" and move closer to a more human way of processing information and therefore to a more natural feeling in the synchronous conversations sustained between these systems and their users.

This is especially noticeable in the case of online bots, which can interact through typed dialogues with users (especially customers in shopping websites) and access a lot of background information available online in order to adapt the conversation to the user's needs. Adams (2018) defines bots as any automated process present in a computer network that mimics online human behaviour, and these have become highly "human" in their ability to match the user's linguistic input with background contextual information in order to generate relevant utterances to the users and relevant interpretations from the user's utterances. These bots rely on algorithms and access to big data accumulated on the website (see previous Section) in order to provide users with the expected answers and suggestions.

\section{Social networking sites and identity-shaping}


Social networking sites such as Facebook or Instagram are basically intended to offer users an area for self-expression, identity shaping and display of interactions that produce effects on the user's personal and social identities. These sites are bound to remain important in the future and to be an inherent object of research for internet pragmatics.

As is summarised in Yus (2018d), users shape their identities on these sites by means of different types of uploaded (verbal-visual-multimodal) discourse, users position themselves as unique individuals and upload content on their profiles with expected audiences and interpretations, a kind of identity performance; and thanks to the affordances of new media, "addressee users" also co-construct, co-produce text in a joint generation of content.

However, as has been argued in Yus (2018e), if we base the eventual relevance of internet communication only on the objective interest and value aroused by the intentional propositional content uploaded and transferred to other users, we will be unable to explain much of the appeal and the specificity of the Net in terms of user satisfaction and engagement (and eventual impact on the user's self-image). Certainly, as was briefly provided in Section 7 above, users often not only obtain relevance from the information provided by the content itself, but also (and nowadays especially) from the feelings and emotions that this content produces in addressee users, often leaking beyond the interlocutors' awareness. The kind of effect that is important in this case is the one which exhibits a non-intentional and non-propositional quality, sometimes adding to the relevance of the propositional information and, crucially, often making up for the objective irrelevance of the information contained in the discourse communicated.

The eventual relevance of internet-mediated acts of communication on social networking sites may be affected by the user's attributes and motivations, especially the user's personality and how it is managed in the online/offline interface. Users frequently present an enhanced version of themselves and the act of presentation, even if faithful to the user's offline identity, is always constrained (qualitatively and quantitatively) by the user's personality, self-esteem, strength of ties, feelings and emotions. Besides, the user's uploaded content is also constrained by general expectations of conformity to norms and group-related expectations. This is especially the case of young users, always eager to find ways to leave a positive impression on their audiences and in the process they are constantly monitoring what their peers expect from them.

Furthermore, as was argued in Yus (2018f), identity on social networking sites demands the cross-breeding of different disciplines since it is a highly complicated phenomenon. Take, for instance, what in Yus (2016c) was labelled correlation of an identity-centred act of communication, exemplified in the one described in (9), and which, in this case, is made up of four phases:

(9) a. A user with a narcissistic personality and a command of how to use interfaces.

b. The user uploads personal photos, pics of trips, etc. on Facebook, aiming at an impact.

c. The user gets "likes" and praising comments. Interactions are triggered.

d. The user gets an (un)conscious offset of self-affirming positive effects (emotions).

In this case, phase (9a) would be treated as a user-related contextual constraint, specifically regarding the user's personality, self-concept and identity. This should be analysed by psychology or sociology, among other possible disciplines. Phase (9b) would be considered an interface-related constraint, associated with overall system usability (i.e. interface affordances) and options for contextualisation and user expression, mainly studied by computer science. Besides, visual discourses on the page would be addressed, for instance, by semiotics, whereas 
the text on the page would be a more appropriate object for internet pragmatics. Phase (9c), based on the production and interpretation of discourse, would typically be analysed by (cyber)pragmatics, digital discourse analysis, sociolinguistics, etc. Phase (9d) would fit the label of user-centred non-propositional effects, not typically analysed by pragmatics (but starting to arouse its interest), so they would be analysed by psychology, or even sociology (group-related effects). Apart from these phases, in this correlation there would also be macro-social contextual constraints, in the sense that the production and reception of content is tied to a culture and its specificity, for example in the individualist/collectivist cultural dichotomy, problems with cultural censorship, etc., aspects typically addressed by sociology, ethnography, cultural studies, etc.

\section{Online polylogues}

In the future, internet pragmatics will mainly analyse online interactions that are increasingly devoid of the traditional dyadic quality, that is, utterances which are no longer exchanged between one single sender and one single receiver. This traditional object of pragmatic research is now more frequently multi-party and forms different layers of interaction or polylogues, either text-based (Section 3 above) or with the aid of visual and multimodal content (Section 2 above) or even with the mediation of voice (Section 4 above). For example, Bou-Franch et al. (2012) analyse how YouTube interactions constitute online polylogues or multi-participant interactions where users can participate on two levels: on the one hand, users may initially contribute actively to the textual polylogues; on the other, they can also participate passively, without posting audiovisual and/or textual responses. When labelling users as "active," they refer to the owner of the channel and registered users, while passive participants are (non)registered users who watch the video-clip and/or read the comments. Different participants in the polylogue have access to different additional communicative actions (see also Marcoccia 2004, Lorenzo-Dus et al. 2011, Bou-Franch and Garcés-Conejos Blitvich 2014, and Bondi 2018).

\section{Media convergence and multiple simultaneous areas of interaction}

New interfaces for interaction are becoming popular on the internet. Some of these include different areas for interaction which have different options for contextualisation and different speeds for production and interpretation of the messages. Internet pragmatics will find an interesting object of research in these new interfaces.

A good example of these interfaces that contain different areas for interaction (and which also entail different modes of interaction) is to be found in the sites for live streaming such as Twitch (Figure 4). In a nutshell, what interests internet pragmatics most is not so much the fact that live streaming links the physical and the virtual (i.e., scattered users watching live what someone is doing in a physical scenario), but the fact that interfaces for live streaming exhibit an interesting media convergence. Indeed, live streaming platforms often combine the actual video being recorded, but also a text-based chat application, possibility of synchronous video interaction, a possibility of commenting on videos or games being played, plus profiles similar to those on social networking sites, together with innovative ways in which content is commented upon, shared and communally enhanced. 


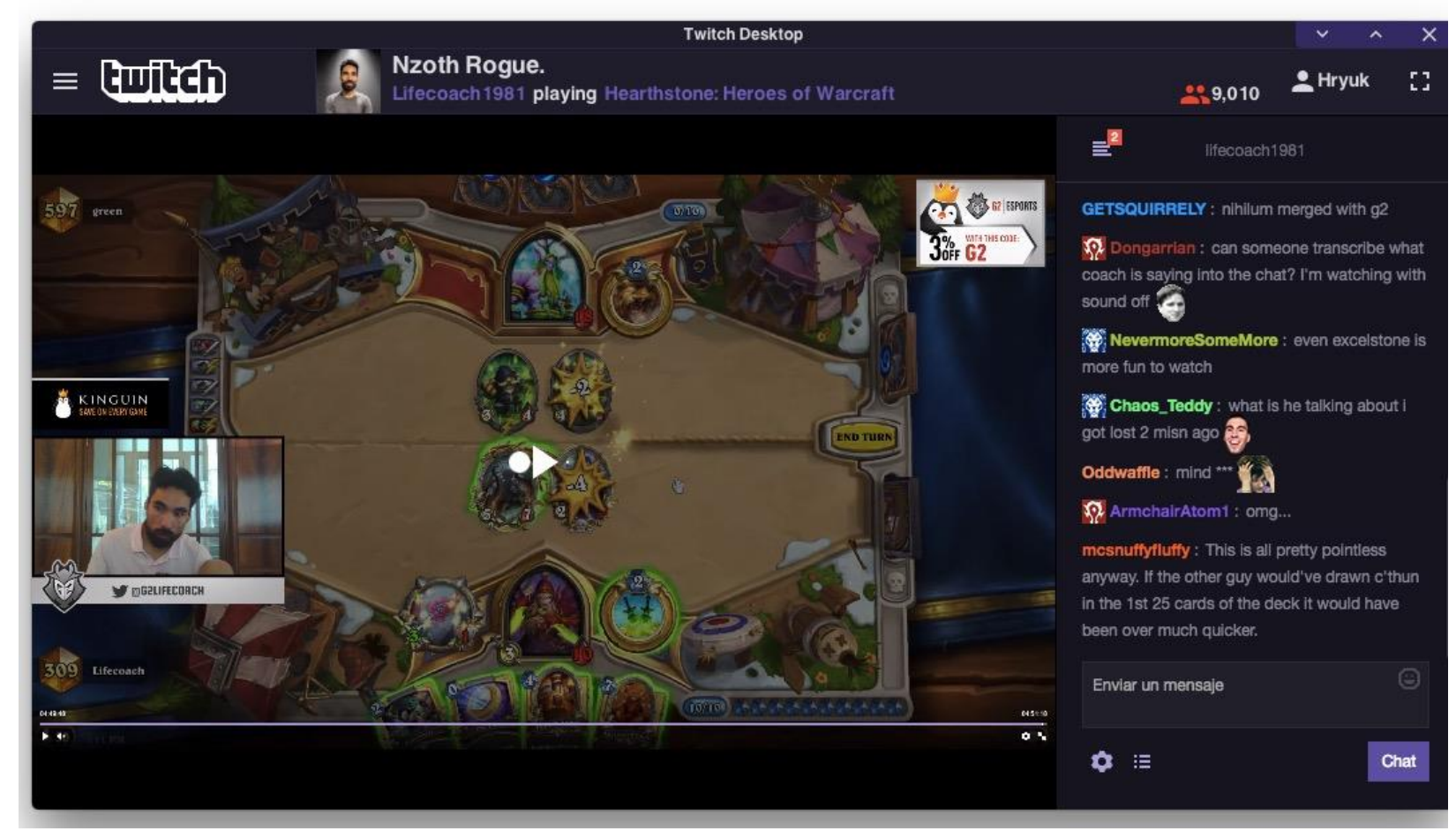

Figure 4. Twitch's desktop interface.

Twitch combines certain features of social networking sites plus video-mediated communication and a text-based chat affordance, so all kinds of overlapping interactions with different communicative speeds and options for contextualisation take place in these environments, a very attractive object of research for internet pragmatics. These interactions may be quite complex. Viewers can send text messages to other viewers and the streamer as a communication backchannel among the participants. Those text messages are integrated with the display of the accompanying video, and are often used to express reactions, add commentary, and even make requests to the broadcasting streamer. Since all the viewers can see these chat messages, they often use it to communicate with each other by building on others' reactions or answering each other's questions. Streamers monitor the text chats as well, but typically speak their responses into the live stream rather than type into the chat (Miller et al. 2017: 2394). Besides, video game streaming has brought back some of the social interaction that had been absent from the domain of video games. Therefore, we are not only witnessing a convergence of interactive and "passive" media but also aspects of the media experience come and go depending on the platforms on which they are consumed (Sjöblom et al. 2017: 168), also an interesting topic for internet pragmatics.

\section{Blurring of traditional elements in communication (author, discourse, audience)}

Pragmatics has typically analysed a clear-cut communicative situation between one addresser, who intentionally produces a piece of discourse (or nonverbal behaviour) to be interpreted by an audience. The focus is mainly on analysing the online discourse itself in terms of usability and options for contextualisation. This discourse provides analysts with an evidence of underlying intentions, of the sender's expectations of relevance, of expected effects on addressee users, of intended interpretation(s) arising from initial decoding of the schematic discourse that is coded (typed, said), and of the expected mutuality of information. Furthermore, the discourse produced 
allows us to foresee how the addressee user will turn what he/she reads/hears into a relevant interpretation, including possible effects the discourse may generate in the addressee user beyond discourse interpretation.

This prototypical communicative schema is no longer the norm nowadays, thanks to the affordances of internet interfaces and the new ways of generating content that the internet offers users. This reinterpretation of the schema will suit some users (accustomed to more dynamic forms of interaction, who like to take full responsibility in the act of communication and its production and/or interpretations), while discouraging other users (who prefer a more traditional way of interacting and of processing content). As proposed in Yus (2015b) concerning new narratives but equally applicable to any type of online interaction, with the rise and ubiquity of the net, what we rather witness nowadays is: (1) new senders/authors (collective creation, hybridization of sender-addressee, etc.); (2) who produce new forms of discourse (audio-visual, multimodal discourses, link-mediated choices for the flow of the text, etc.); (3) through new interfaces (new verbal-visual designs, multimodality, interfaces aiming at usability); (4) aimed at a new kind of addressee (an active, dynamic one who often contributes to the authorship of the discourse); and (5) who comes up with a typology of interpretations (the sender's intended interpretation -if any- is diluted and the choice of interpretations is mainly the addressee's responsibility). These are briefly commented upon below.

(1) New senders/authors... The makers of online discourses no longer expect a specific interpretation, nor do they expect that their readers will invariably follow certain reading paths (e.g. links) in a certain order. Besides, their presence in the discourse -for example as narratorsis weaker and less visible, as if only making information manifest to other users was their sole task in this typed (or recorded) mode of communication. Besides, readers are very active in processing digital discourses, to the extent that they even become co-participants in the authorship. This has a "diluting effect" in terms of what specific interpretation -if any- underlies the act of creation of that discourse.

(2) ... who produce new kinds of discourse... The traditional cumulative flow of information (in which new information is coherently added and becomes a preliminary context for subsequent processing of information) collapses completely with new discourses and levels of reader involvement that will appeal to certain users (those internet users who are keen on discursive innovations) while dissuading others (older users accustomed to a traditional cumulative flow of information).

(3) ...through new interfaces... The discourse itself undergoes major alterations in this new online scenario. On the one hand, online discourses are no longer strictly text-based, but are complemented with images, video, links, animated graphs, multimodal compositions, etc. Traditional textual discourses have turned into transmedial or multimodal ones. Therefore, the source of meaning often lies in the combination of information provided by different types of media involving interpretive choices by the user. Besides, it is also very important to consider how the interfaces are designed for usability, with menus, screens and links that should lead readers in the right direction (or should make it easier for the reader to take any direction he/she wants) without an increase in mental effort generated by the use of the interface.

(4) ... aimed at a new kind of reader. Today's user is no longer a passive addressee of delimited and unchangeable texts. Rather, these readers are active, participatory, co-creators of the discourse and willing to take full responsibility for which links they click on, in which order, and for which coherence they apply to the subsequent nodes they access after having decided on which links to click on (Storrer 2002). This constant choice of links to click on and the need to find inter-link coherence reduce the user's attention that is available for text comprehension, which may be another possible instance of a negative contextual constraint. 
In this sense, the typical reader of today's social media is different from the one in traditional media communication, as is described in Alexander and Levine (2008: 47). For these authors, user-generated content is a key element of today's web 2.0. A reader can add content into story platforms directly: editing a wiki page, commenting on a post, replying in a Twitter feed, posting a video response in YouTube. Those interactions fold into the experience of the overall story from the perspective of subsequent readers. Today's online stories tend to be accretions over time, imbricated layers of content on top of an original core.

(5) Who come up with a typology of interpretations. In this scenario of new types of discourse in which the traditional roles of author and reader are diluted and in which there are so many options to click on, there is no prediction of what information will be more relevant to the actual reader or hearer, or even what the exact (i.e. intended) interpretation might be. It all depends on the actual context of interpretation, unique in every situation. Different readers with different backgrounds and context accessibility will deliver radically different interpretations of the same discourse.

\section{Interactions in scenarios where a virtual layer of information is superimposed on the physical}

Locative media have produced a layer of information that users superimpose on physical locations. This information generates different areas of mutuality and different conceptualisations of space according to the users' comments. However, there is another possible layer of information superimposed on the physical which is not managed by the users, but by the companies making mobile interfaces and apps that provide users with supplementary information related to the place where the user is located or about the user's whereabouts. One of the terms fitting this additional layer is augmented reality. Again, in a similar way to what is at stake in the case of locative media, this layer of supplementary information will have an impact on what information is shared between users, on which area of this layer is found relevant, and especially on how this layer may contribute to forming preliminary contexts upon which subsequent virtual interactions may be sustained. Internet pragmatics will probably have interesting future research in this area.

\section{Need of ethnographic approaches and analyses of data, for example on Facebook}

In the future, internet pragmatics will tend to rely on ethnographic analyses of data, for example entries and comments of Facebook or conversations in mobile instant messaging interactions, in which the user gets "an insider's view" of interactions and communication (see Pink et al. 2016, Hjorth et al. 2017, among others). For that purpose, there is now a wide range of software applications that aid the analyst in gathering and tagging data according to some research parameters.

\section{Cross-cultural issues}

The Internet is a typically globalised phenomenon, with companies such as Facebook that have millions of users scattered all over the world. However, very often the interfaces and applications are specially tailored to each specific culture, and even if the interface is the same 
across cultures, as happens with Instagram or Facebook, culture-specific uses of these platforms abound, and internet pragmatics will remain interested in differentiating these culture-connoted discursive uses. Among other research issues, internet pragmatics will be interested in the discursive impact of individualist versus collectivist cultures, in how different types of interactions are biased by cultural protocols and principles, in how, in some cultures, access to information and the use of certain interfaces are limited and controlled by authorities (censorship), and in how the oralised typed text (i.e. text deformation) and the use of emoji are also culturally biased, including culture-specific sets of textual strategies and galleries of emoji, among other interesting culture-specific research topics.

\section{Concluding remarks}

In this paper a review has been proposed of several research issues that, in my opinion, are bound to be important objects of analysis by internet pragmatics in the near future. Initially, the main objects of pragmatic research offline, such as the analysis of what is said versus what is meant (and interpreted) (Section 1), the need of ethnographic approaches and analyses of data (Section 17) and the existence of cross-cultural issues in communication (Section 18) will also be applicable to online interactions but necessarily taking into account the specificity of the interfaces through which these interactions are managed. For example, voice-based communication on mobile phone apps should be similar to face-to-face interactions, but these exhibit several specificities (Section 4), and the same applies to the depth and extent of information shared between interlocutors in overlapping online/offline scenarios (Section 6). Furthermore, interfaces today and in the future tend to contain different media and multiple simultaneous areas of interaction, each of them with different speeds and interpretive constraints, as happens with sites for video streaming such as Twitch (Section 14).

Similarly, typed messages in messaging applications have to be analysed together with the specific options for visual support of the app (emoji, GIFs, stickers) and the ability to convey vocal qualities of typed text through so-called text deformation (Section 3). In general, it can be stated that most online discourses nowadays have to be analysed in the combination of visual and verbal inputs in mainly multimodal instances of communication (Section 2). And the analysis of interactions will have to move beyond the prototypical dyadic structure into the more pervasive format of polylogue (Section 13) and with a clear blurring of traditional elements in communication such as author, discourse and audience (Section 15). This is also the case of social networking sites (e.g. Facebook, Instagram) and how users upload multimodal content in their profiles mainly for identity-shaping and self-expression purposes (Section 12).

Future internet pragmatic research will also have to focus on some puzzling aspects of today's online interactions. For example, users were in the past liberated from the need to interact from a specific location thanks to the development of mobile phones, but now the physical place has become important in internet communication and users are again tied to a specific location (Section 5). A similar puzzling aspect is the decreasing importance of substantive content in exchange for phatic interactions on the Net. This may be explained thorough two new notions that have been added to the overall pragmatic (and cyberpragmatic) research: contextual constraints (Section 8) and non-intended non-propositional effects (Section 9).

Finally, internet pragmatics will also be interested in borderline areas of online communication that involve human users and computer systems. Big data will no doubt have an impact on the quality and quantity of online interactions and the amount of information shared 
between interlocutors (Section 10). Besides, virtual agents and bots will increasingly exhibit human attributes and will be able to engage in interactions in a human-like way (Section 11), thus deserving pragmatic analysis. Finally, internet pragmatics will also become interested in interactions where a virtual layer of information is superimposed on the physical, as happens with augmented reality (Section 16).

\section{References}

Adams, Tim. 2018. "How can I tell if I am talking to a real person online?" The Guardian, November 18, 2018. The New Review, p. 12-15.

Alexander, Brian, and Alexander Levine. 2008. "Storytelling. Emergence of a new genre." Educause Review 43(6): 40-56.

Bondi, Marina. 2018. "Blogs as interwoven polylogues. The dialogic action game." Language and Dialogue 8(1): 43-65.

Bou-Franch, Patricia, Nuria Lorenzo-Dus, and Pilar Garcés-Conejos Blitvich. 2012. "Social interaction in youtube text-based polylogues: A study of coherence." Journal of Computer-Mediated Communication 17: 501-521.

Bou-Franch, Patricia, and Pilar Garcés-Conejos Blitvich. 2014. "Conflict management in massive polylogues: A case study from YouTube.” Journal of Pragmatics 73: 19-36.

Carr, Caleb T., D. Yvette Wohn, and Rebecca A. Hayes. 2016. "[Like] as social support: Relational closeness, automaticity, and interpreting social support from paralinguistic digital affordances in social media." Computers in Human Behavior 62: 385-393.

Dayter, Daria. 2016. Discursive Self in Microblogging. Speech Acts, Stories and Self-praise. Amsterdam: John Benjamins.

Grice, Herbert P. 1975. "Logic and conversation." In Speech Acts (Syntax and Semantics 3), ed. by P. Cole, and J. Morgan, 41-58. New York: Academic Press.

Flores Salgado, Elizabeth, and Teresa A. Castineira-Benitez. 2018. "The use of politeness in WhatsApp discourse and move 'requests'." Journal of Pragmatics 133: 79-92.

Forceville, Charles, and Billy Clark. 2014. "Can pictures have explicatures?" Linguagem em (Dis)curso (special issue on relevance theory) 14(3): 451-472.

Frith, Jordan. 2015. Smartphones as Locative Media. Cambridge: Polity.

Grundlingh, L. 2018. "Memes as speech acts." Social Semiotics 28(2): 147-168.

Hjorth, Larissa, Heather Horst, Anne Galloway, and Genevieve Bell. eds. 2017. The Routledge Companion to Digital Ethnography. Abingdon: Routledge.

Jewitt, Carey. 2016. "Multimodal analysis." In Handbook of Language and Digital Communication, ed. by Alexandra Georgakopoulou \& Tereza Spilioti, 69-84. Abingdon: Routledge.

Langlotz, Andreas, and Miriam Locher. 2013. "The role of emotions in relational work." Journal of Pragmatics 58: 87-107.

Licoppe, Christian. 2013. "Merging mobile communication studies and urban research: Mobile locative media, "onscreen encounters" and the reshaping of the interaction order in public spaces." Mobile Media and Communication 1(1): 122-128.

Lorenzo-Dus, Nuria, Pilar Garcés-Conejos Blitvich, and Patricia Bou-Franch. 2011. "On-line polylogues and impoliteness: The case of postings sent in response to the Obama Reggaeton YouTube video." Journal of Pragmatics 43: 2578-2593.

Lutzky, Ursula, and Matt Gee. 2018. "I just found your blog'. The pragmatics of initiating comments on blog posts." Journal of Pragmatics 129: 173-184.

Marcoccia, Michel. 2004. "On-line polylogues: conversation structure and participation framework in internet newsgroups." Journal of Pragmatics 36: 115-145.

McKeown, Jamie, and Qilin Zhang. 2015. "Socio-pragmatic influence on opening salutation and closing valediction of British workplace email." Journal of Pragmatics 85: 92-107.

Miller,Vincent. 2008. "New media, networking and phatic culture." Convergence 14: 387-400. 
Miller, Matthew K., John C. Tang, Gina Venolia, Gerard Wilkinson, and Kori Inkpen. 2017. "Conversational chat circles: Being all here without having to hear it all." In Proceedings of CHI 2017. Denver, CO, USA, 2394-2404.

Morrow, Philip R. 2017. "Requesting and advice giving." In Pragmatics of Social Media, ed. by C. R. Hoffmann and W. Bublitz, 661-689. Berlin/Boston: De Gruyter Mouton.

Pariser, Eli. 2011. The Filter Bubble. What the Internet is Hiding from You. New York: Penguin Press.

Paulus, Trena, AmberWarren, and Jessica Nina Lester. 2016. "Applying conversation analysis methods to online talk: A literature review." Discourse, Context and Media 12: 1-10.

Pink, Sarah, Heather Horst, John Postill, Larissa Hjorth, Tania Lewis, and Jo Tacchi. 2016. Digital Ethnography. Principles and Practice. London: Sage.

Placencia, María Elena, Amanda Lower, and Hebe Powell. 2016. "Complimenting behaviour on Facebook. Responding to compliments in American English." Pragmatics \& Society 7(3): 339-365.

Sampietro, Agnese. 2016. "Exploring the punctuating effect of emoji in Spanish WhatsApp chats." Lenguas Modernas 47: 91-13.

Schubert, Christoph. 2017. "Discourse and cohesion." Pragmatics of Social Media, ed. by C. R. Hoffmann and W. Bublitz, 317-343. Berlin/Boston: De Gruyter Mouton.

Schwartz, Raz. 2015. "Online Place Attachment: Exploring Technological Ties to Physical Places." In Mobility and Locative Media. Mobile Communication in Hybrid Spaces, ed. by Adriana de Souza e Silva, and Mimi Sheller, 85-100. Abingdon: Routledge.

Scott, Kate. 2018. "The pragmatics of hashtags: Inference and conversational style on Twitter." Journal of Pragmatics 81: 8-20.

Sjöblom, Max, Maria Törhönen, Juho Hamari, and Joseph Macey. 2017. "Content structure is king: An empirical study on gratifications, game genres and content type on Twitch." Computers in Human Behavior 73: 161-171.

Sperber, Dan, and Deirdre Wilson. 1995. Relevance: Communication and Cognition (2nd edition). Oxford: Blackwell.

Stöckl, Hartmut. 2004. "In between modes: Language and image in printed media." In Perspectives on Multimodality, ed. By Eija Ventola, Cassily Charles \& Martin Kaltenbacher, 9-30. Amsterdam: John Benjamins.

Storrer, Angelika. 2002. "Coherence in text and hypertext." Document Design 3(2): 156-168.

Turkle, Sherry. 2015. Reclaiming Conversation. The Power of Talk in a Digital Age. New York: Penguin Press.

van Kleef, Gerben A. 2016. The Interpersonal Dynamics of Emotion. Toward an Integrative Theory of Emotions as Social Information. Cambridge: Cambridge University Press.

van Leeuwen, Theo. 2015. "Multimodality." In The Handbook of Discourse Analysis (2nd edition), ed. By Deborah Tannen, Heidi E. Hamilton \& Deborah Schiffrin, 447-465. Oxford: Wiley Blackwell.

Vetere, Frank, Jeremy Smith, and Martin Gibbs. 2009. "Phatic interactions: Being aware and feeling connected." In Awareness Systems. Advances in Theory, Methodology, and Design, ed. by Panos Markopoulos, Boris de Ruyter, and Wendy Mackay, 173-186. Berlin: Springer.

Wang, Victoria, John V. Tucker, and Tracey E. Rihll. 2011. "On phatic technologies for creating and maintaining human relationships." Technology in Society 33(1-2): 44-51.

Wilson, Deirdre. 2017. "Communication, comprehension and 'non-propositional' effects." Paper delivered at Beyond Meaning. Athens (Greece), September.

Wiseman, R. 2013. "What boys want". Time, 2-12-2013, pp. 24-31.

Yus, Francisco. 1998. "A decade of relevance theory.” Journal of Pragmatics 30: 305-345.

Yus, Francisco. 2005. "Attitudes and emotions through written text: The case of textual deformation in Internet chat rooms." Pragmalingüística 13: 147-174.

Yus, Francisco. 2008. "Inferring from comics: A multi-stage account." Quaderns de Filologia. Estudis de Comunicació III: 223-249.

Yus, Francisco. 2010. "Relevance theory." In The Oxford Handbook of Linguistic Analysis, ed. by B. Heine, and H. Narrog, 679-701. Oxford: Oxford University Press.

Yus, Francisco. 2011a. Cyberpragmatics. Internet-Mediated Communication in Context. Amsterdam and Philadelphia: John Benjamins. 
Yus, Francisco. 2104. “"Not all emoticons are created equal.” Linguagem em (Dis)curso (special issue on relevance theory) 14(3): 511-529.

Yus, Francisco. 2015a. "Discourse and identity." In: International Encyclopedia of the Social \& Behavioral Sciences (2nd edition). Volume 6, ed. by J.D. Wright, 498-502. Oxford: Elsevier.

Yus, Francisco. 2015b. "The role of cognition and relevance in new digital narratives." Prospettive multilingue e interdisciplinari nel discorso specialistico, ed. by E. Carpi, 81-107. Pisa: Pisa University Press.

Yus, Francisco. 2016a. "Discourse, contextualization and identity shaping. The case of social networking sites and virtual worlds." In New tools, New Approaches. Technology implementation in Higher Education for Second Language Teaching and Translation Studies, ed. by M.L. Carrió-Pastor, 7188. Berlin: Springer.

Yus, Francisco. 2016b. Humour and Relevance. Amsterdam: John Benjamins.

Yus, Francisco. 2016c. "Online identity: A (non)propositional account." Paper delivered at EPICS VII. Pablo de Olavide University (Seville), May.

Yus, Francisco. 2017a. "Contextual constraints and non-propositional effects in WhatsApp communication." Journal of Pragmatics 114: 66-86.

Yus, Francisco. 2017b. "Putting relevance at centre stage in research on human activity on the Internet." In Applications of Relevance Theory: From Discourse to Morphemes, ed. by Agnieszka Piskorska, and Ewa Walaszewska, 86-102. Newcastle-upon-Tyne: Cambridge Scholars Publishing.

Yus, Francisco. 2018a. "The interface between pragmatics and Internet-mediated communication: Applications, extensions and adjustments. In Pragmatics and its Interfaces, ed. by C. Ilie, and N. Norrick, 267-290. Amsterdam: John Benjamins.

Yus, Francisco. 2018b. "Multimodality in memes. A cyberpragmatic approach." In Analyzing Digital Discourse: New Insights and Future Directions, ed. by Patricia Bou-Franch, and Pilar GarcésConejos Blitvich, 105-131. Cham (Switzerland): Palgrave Macmillan.

Yus, Francisco. 2018c. "Cyberpragmatics of interactions through locative media." Paper delivered at International CoCoLaC-Conference. Comparative Approaches to Pragmatics. Helsinki, March.

Yus, Francisco. 2018d. "Attaching feelings and emotions to propositions. Some insights on irony and internet communication." Russian Journal of Linguistics 22(1): 94-107.

Yus, Francisco. 2018e. "Relevance from and beyond propositions. The case of online identity. In Relevance and Irrelevance: Theories, Factors and Challenges, ed. by H. Nasu, and J. Strassheim, 119-140. Berlin: De Gruyter.

Yus, Francisco. 2018f. "The future of internet pragmatics." Paper delivered at First International Conference on Internet Pragmatics. Fuzhou (China), September.

Yus, Francisco. in press. "A cognitive pragmatics of the phatic Internet." In Emotion in Discourse, ed. by Laura Alba, and Lachlan Mackenzie, 161-188. Amsterdam: John Benjamins.

Žegarac, Vladimir. 1998. "What is 'phatic communication'? In Current Issues in Relevance Theory, ed. by Villy Rouchota and Andreas H. Jucker, 327-362. Amsterdam: John Benjamins.

Zhao, Shanyang, Sherri Grasmuck, and Jason Martin. 2008. "Identity construction on Facebook: Digital empowerment in anchored relationships." Computers in Human Behavior 24: 1816-1836. 